\title{
Facilitation of the main generator source of earthworm muscle contraction by a peripheral neuron
}

Y.C. Chang, Z. Assmé and A.B. Bartoszeck

\author{
Departamento de Fisiologia, U niversidade Federal do Paraná, \\ Curitiba, PR, Brasil
}

\section{Correspondence \\ Y.C. Chang \\ Departamento de Fisiologia Setor de Ciências Biológicas, UFPR \\ Caixa Postal 8621 \\ 80011-970 Curitiba, PR \\ Brasil \\ Fax: + 55-41-266-2042 \\ E-mail: changyc@cce.ufpr.br \\ Part of a Doctoral thesis presented by $Z$. Assmé to the Departamento de Fisiologia, UFPR, Curitiba, PR, Brasil.}

Received January 20, 1997 Accepted July 27, 1998

\section{Abstract}

A constant facilitation of responses evoked in the earthworm muscle contraction generator neurons by responses evoked in the neurons of its peripheral nervous system was demonstrated. It is based on the proposal that these two responses are bifurcations of an afferent response evoked by the same peripheral mechanical stimulus but converging again on this central neuron. A single-peaked generator response without facilitation was demonstrated by sectioning the afferent route of the peripheral facilitatory modulatory response, or conditioning response (CR). The multipeaked response could be restored by restimulating the sectioned modulatory neuron with an intracellular substitutive conditioning stimulus (SCS). These multipeaked responses were proposed to be the result of reverberating the original single peaked unconditioned response (UR) through a parallel (P) neuronal circuit which receives the facilitation of the peripheral modulatory neuron. This peripheral modulatory neuron was named "Peri-Kästchen" (PK) neuron because it has about 20 peripheral processes distributed on the surface of a Kästchen of longitudinal muscle cells on the body wall of this preparation as revealed by the Lucifer Yellow-CH-filling method.

\section{Introduction}

After the main generator source of effector muscle contraction of the earthworm, Amynthas hawayanus, was identified as an M (mechanical-stimulus-sensitive) circuit composed of two neurons (1), the purpose of this second paper was to identify the mechanism by which the responses of this main generator source is influenced by another circuit in its epidermis-muscle-nerve-cord preparation, resulting in the modified effector muscle contraction magnitude (2). Neu-

\section{Key words}

- Peri-Kästchen neuron

- Facilitation of main generator response responses
- Single and multipeaked 
M-circuit and one of the INT neurons (here designated as "Peri-Kästchen" (PK) neuron, see explanation below) was proposed in a diagram (Figure 1) based on the fact $(1,3)$ that responses in both circuits were evoked by the same mechanical stimulus (MS; Figure 1). The afferent pathway from the same mechanoreceptor (S; Figure 1) was proposed to bifurcate into the UR (Figure 1) and the CR (Figure 1). This proposal of bifurcated afferent pathways was used as a guide for the identification of this mechanism in the present article and for comparison of mechanisms in the third article (4). However, if the UR and CR are assumed to be simultaneous, they cannot be independently manipulated in experiments such as varying the interstimulus interval (ISI) between them. The natural CR in the INT (or PK) neuron

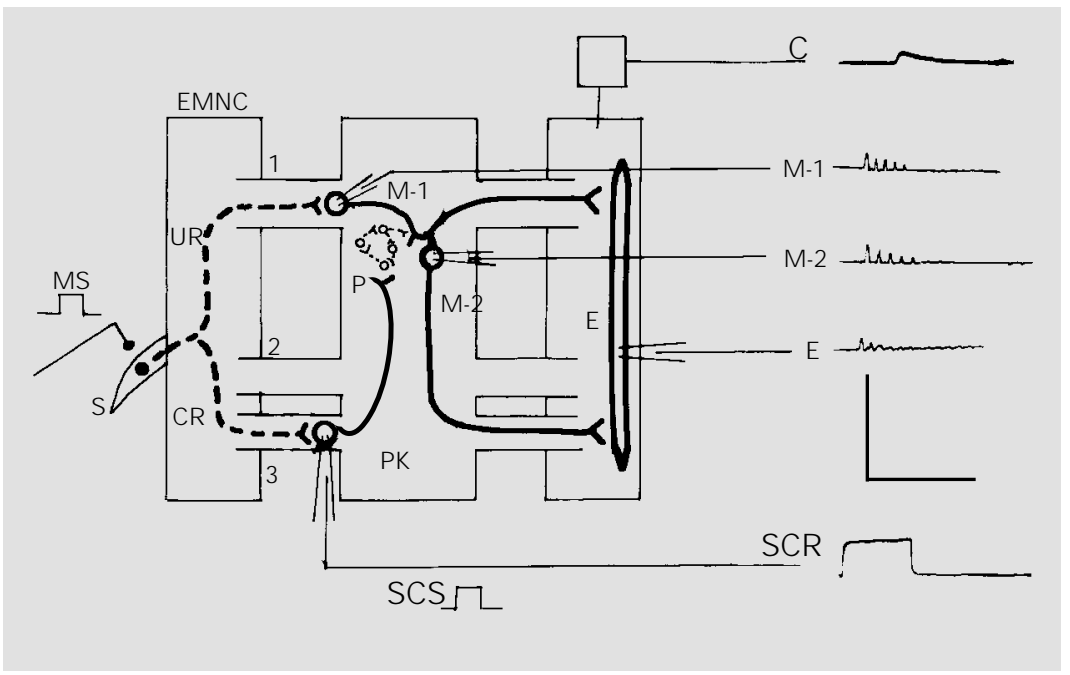

Figure 1 - Proposed neuronal circuit and their responses. EMNC, Epidermis-muscle-nervecord preparation with the anterior end at the top of the figure. The two lateral columns are the partitioned epidermis-muscle pieces. The central column is the ventral nerve cord. Three pairs of intact segmental nerves, with numbers, connect the two epidermis-muscle pieces to the ventral nerve cord. Solid lines indicate identified neurons and muscle cells. Broken lines indicate still unidentified neurons and routes. Sample responses from these neurons and muscle cells are shown on the right side of the figure. MS, Mechanical stimulus to the setal mechanoreceptor, $\mathrm{S}$, in the left epidermis-muscle piece; UR, unconditioned response; $\mathrm{CR}$, conditioning response; $\mathrm{C}$, muscle contraction. The square symbol is the mechano-electrical transducer. M-1, Presynaptic mechanical-stimulus-sensitive neuron; $M-2$, postsynaptic mechanical-stimulus-sensitive neuron; $P$, parallel neuronal circuit; $E$, effector muscle cell; PK, Peri-Kästchen neuron; SCS, substitutive conditioning stimulus; SCR, substitutive conditioning response. Conventional symbols of circles and lines for cat spinal neurons are used to represent neurons and muscle cells in this figure. Their realistic morphology is shown in Figures 6,7 and 8 of this article. Calibration: for $\mathrm{C}, 5 \mathrm{~g}$ and $5 \mathrm{~s}$; for $\mathrm{M}-1, \mathrm{M}-2, \mathrm{E}$ and SCR, $50 \mathrm{mV}$ and $50 \mathrm{~ms}$; for SCS, $50 \mathrm{nA}$ and $50 \mathrm{~ms}$. must be eliminated by differential sectioning (Figure 2) and a substitutive conditioning response (SCR; Figure 1) must be evoked in this neuron by a manipulable substitutive conditioning stimulus (SCS; Figure 1).

\section{Material and Methods}

The same epidermis-muscle-nerve-cord preparation (EMNC; Figure 1) of the earthworm, Amynthas hawayanus, illustrated in Figure 2-5 in the first study (1) in this series, with a partitioned epidermis-muscle piece connected to the ventral nerve cord through three pairs of intact segmental nerves, was used in the present study. A mechanical stimulus (Figure 1) was delivered to the setal mechanoreceptor (S; Figure 1) at the partitioned left (stimulated) epidermis-muscle piece. Four microelectrodes with Lucifer Yellow-CH were used to record the responses evoked by this mechanical stimulus in the M-1 and M-2 neurons on the right (recording) side of the nerve cord (2), in the effector muscle cell (E; Figures 1 and 7) and in one of the INT neurons in the left third nerve (3). The postsynaptic response from the M-2 neuron was used as the control response or UR in the present study.

As a first step, it must be demonstrated that only an unconditioned stimulus (US) but not the conditioning stimulus (CS) evokes a UR in the M-2 neuron. This was demonstrated by alternately sectioning the left first (Figure 2-1) and third (Figure 2-2) segmental nerves while the mechanical stimulus was delivered. After eliminating the original CS by sectioning the left third nerve, a substitutive conditioning stimulus SCS (Figure 1) consisting of a 50-ms electrical pulse was delivered to the modulatory neuron ( $\mathrm{PK}$; Figure 3) through the microelectrode in the proximal (cordal) stump of this sectioned left third nerve while leaving the left first nerve intact for delivering the unconditioned stimulus. The effect of this electrical SCS on the modulatory and generator neurons was 
tested by different combinations of polarity, duration and amplitude of this pulse without the unconditioned mechanical stimulus (Figure 3). Restoration of this modulatory facilitation to the unconditioned response was tested by delivering both US and SCS in different ISI to this preparation with a sectioned left third nerve (Figure 4). Summation of the CS and SCS effects on the UR was tested by delivering both stimuli to the intact modulatory neuron without sectioning the left third nerve (Figure 5).

Lucifer Yellow-CH was injected into the generator neurons, modulatory neuron and the effector muscle cell while stimulating and recording. The morphology of the identified modulatory neuron was photographed and camera-lucida drawn in three dimensions (3) (Figure 6). This neuron was tentatively named the Peri-Kästchen (PK) neuron by its morphology (see Results). Its anatomical position relative to the generator M-neurons and to the effector muscle cell was photographed in the radial dimension (PK, Figure 7). Its morphological relation to other intramural, or intermuscular, neurons in this preparation was demonstrated (Figure 8) by replicating the histological method used in references 5-7 based on hematoxylin-eosin staining and paraffin embedding.

\section{Results}

\section{Characteristics of the main generator source response}

After sectioning the left first nerve (Figure 2-1) which contains the afferent process (A-1) of the main generator source (1), the five-peaked responses were no longer recorded from the M-1 and M-2 neurons although the afferent response evoked by the mechanical stimulus was continuously recorded from the PK neuron in the intact third nerve. This experiment showed that the UR was evoked only by the US but not by the bifurcated branch of the CS in the PK neu- ron. On the other hand, section of the left third nerve while leaving the left first nerve intact (Figure 2-2) eliminated the four subsequent peaks while leaving the first peak intact in the responses recorded from the M-1 and M-2 neurons. This experiment suggests that only the first peak was the response evoked in the generator source by the US alone while the four subsequent peaks were the result of facilitation by the modulatory PK neuron. This suggestion will be further tested in the following sections.

\section{Effect of SCS on the generator and modulatory neurons}

The simultaneous occurrence of CS and US was inconvenient for further study. The natural CS was always eliminated by sectioning of the left third nerve and a more controllable SCS (Figure 3) was always delivered to the PK neuron. The effect of this SCS on both the generator (M-2; Figure 3) and modulatory (PK; Figure 3 ) neurons without the mechanical US was tested in 44 preparations. Consistent results were ob-

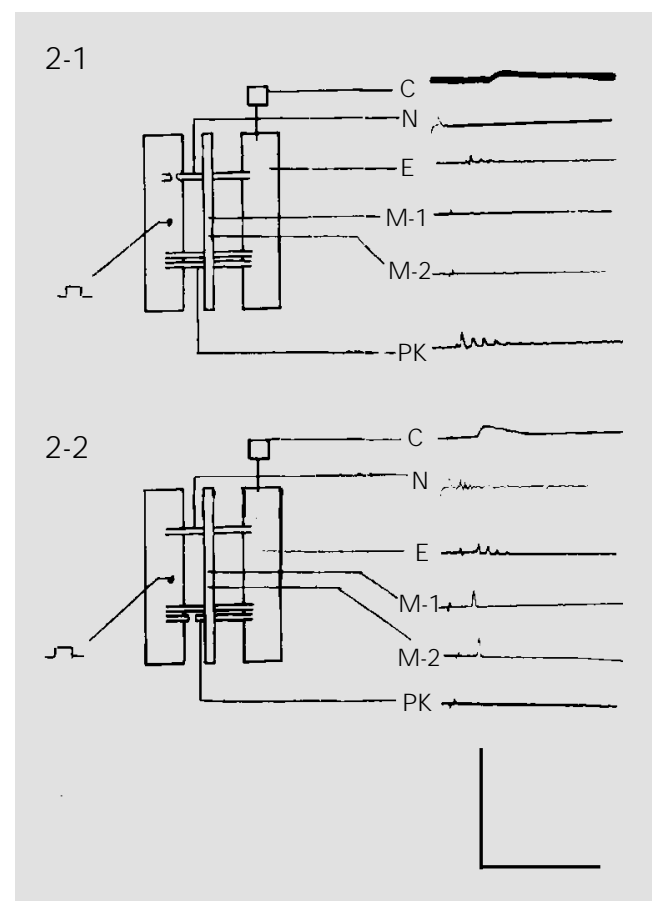

Figure 2 - Elimination of UR peaks.

2-1, Elimination of all peaks by sectioning the left first nerve.

2-2, Elimination of four subsequent peaks by sectioning the left third nerve.

C, Muscle contraction recorded by transducer (square); $\mathrm{N}$, nerve response recorded by suction electrodes; M-1, M-2, E and PK recorded by microelectrodes. Same abbreviations and calibrations as in the legend to Figure 1. 
Figure 3 - Effects of SCS on both generator and modulatory neurons. The diagram at left shows the experimental setup. A microelectrode was inserted into an $\mathrm{M}-2$ neuron only for response recording. Another microelectrode was inserted into a PK neuron for delivering the SCS.

3-1, Subthreshold SCSs. No response was observed in M-2 or PK neurons.

3-2, Threshold depolarizing SCS. An on-response is seen in an M2 neuron.

3-3, Action potential in a PK neuron. More on-response is seen in an $\mathrm{M}-2$ neuron.

3-4, Same as in Figure 3-2 (in fast oscilloscope traces).

3-5, Same as in Figure 3-2 (in faster oscilloscope traces than that in Figure 3-4). The two oblique lines are the rising phases of the depolarizing SCS pulses. 3-6, Hyperpolarizing SCS. An offresponse is seen in an $M-2$ neuron.

Calibration: for Figure 3-1, 3-2, 33 and 3-6, ordinate in $2 \mathrm{mV}$ and 2 nA. Abscissa in $40 \mathrm{~ms}$. For Figure 3-4, same ordinate, abscissa in 10 ms. For Figure 3-5, same ordinate, abscissa in $2 \mathrm{~ms}$. Same abbreviations as in Figure 1. No mechanical stimulus.
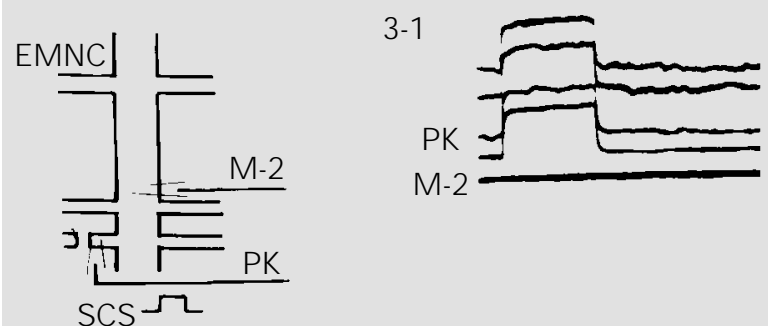

3-4

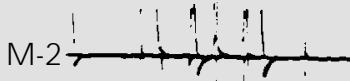

$\operatorname{scs}^{\Omega}$

3-5
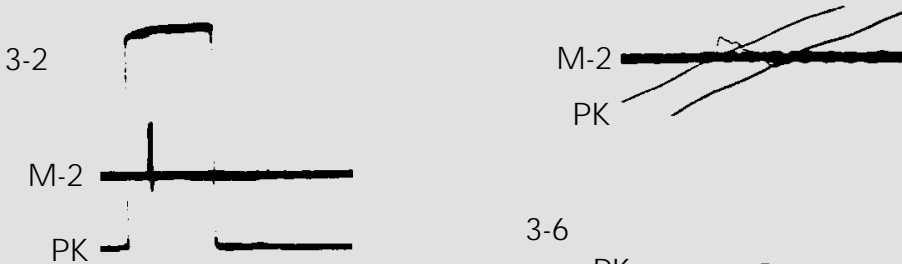

3-3

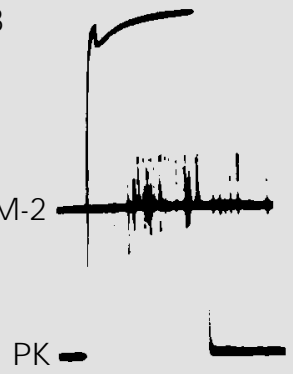

3-6

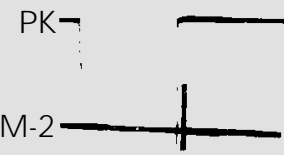

tained in 23 preparations. A series of onresponses was evoked in the generator neurons (M-2; Figure 3-2 and 3-3) when a depolarizing SCS reached 5.0 nA. An action potential was evoked in the modulatory neuron (PK; Figure 3-3) when it reached 7.0 nA while the on-response in the generator neurons continued. When seen in high speed oscilloscope tracings (Figure 3-4 and 3-5), this series of generator neuron on-responses had a time course similar to that of the excitatory postsynaptic potentials (EPSP) (M-2; Figure 3-5). No effector muscle response or contraction was evoked by the action potential in the modulatory neuron or by the EPSP in the generator neuron. Hyperpolarizing SCS evoked no action potential in the modulatory neuron (PK; Figure 3-6) but evoked a series of off-responses, also similar to EPSP, in the generator neurons (M-2; Figure 3-6) when the SCS reached $5.0 \mathrm{nA}$.

\section{Restoration of the four subsequent UR peaks}

After the four subsequent potential peaks in the N-neuron responses were eliminated by sectioning the contralateral (left) third nerve (Figure 2-2) in 15 preparations, they could be restored by restimulating the modulatory PK neuron with a depolarizing SCS (Figure 4). Several conditions must be met in order to restore these subsequent UR peaks. This SCS was not effective in restoring these subsequent peaks if it was delivered with an ISI to the mechanical US. These subsequent peaks could be restored only when the SCS coincided with the US, i.e., ISI $=0$. Only the 
depolarizing SCS (Figure 4-1) could restore these subsequent peaks (M-2; Figure 4-1). The threshold amplitude of this depolarizing SCS for restoring these subsequent UR peaks was around $4.0 \mathrm{nA}$, i.e., lower than its threshold $(7.0 \mathrm{nA})$ of evoking an action potential in the modulatory neuron (PK; Figure 3-3), but similar to that $(5.0 \mathrm{nA})$ for evoking the EPSP in the generator neuron (M-2; Figure 3-2, 3$3)$. A hyperpolarizing SCS of the same amplitude inhibited all peaks including the first in the UR (M-2; Figure 4-2). It inhibited the electrical response in the effector muscle cell (E; Figure 4-2) but did not inhibit the muscle contraction (C; Figure 4-2) of this preparation completely. In 12 of the 15 preparations, not only the subsequent UR peaks were restored by a 4.0-nA depolarizing SCS in the modulatory neuron, but also the first peaks were enhanced to $31.4 \pm 2.5 \mathrm{mV}$, three times their control amplitude (M-2; Figure 4-1). Their effector muscle contraction was also enhanced (C; Figure 4-1), although in an irregular manner.

\section{Summation of the modulatory effects of CS and SCS}

In another 8 of these 23 preparations, the left third nerves were left intact (Figure 5) while both mechanical stimulus and electrical SCS were delivered as in Figure 4. In this case, the original CS was not eliminated because the PK neuron was not sectioned. Its effect was expected to be added to the effect of the SCS. The result was similar to that obtained in the last experiment in Figure 4 except that the UR was more enhanced than by the SCS alone when the depolarizing SCS coincided with the mechanical stimulus (CS and US at the same time) without an ISI. The amplitude of both the first and the subsequent peaks in the UR was enhanced (M-2; Figure 5-1) by a 4.0-nA SCS. The amplitude of the first peaks was enhanced to $48.3 \pm$ $10.5 \mathrm{mV}$ with some maximal enhancements to $60-70 \mathrm{mV}$. Along with the amplitude en-

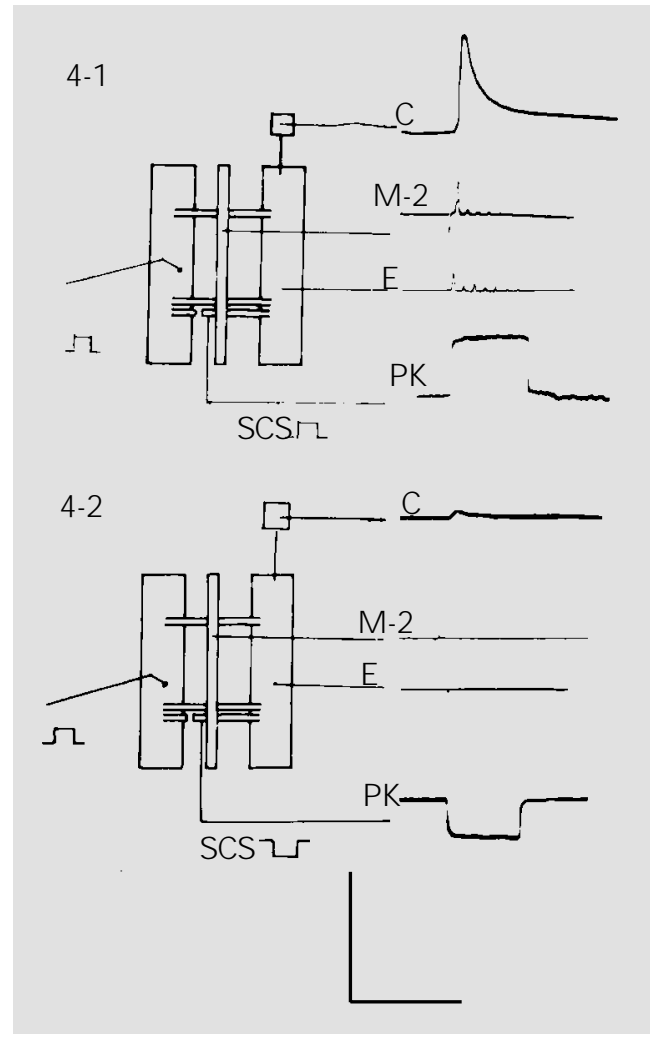

Figure 4 - Restoration of four subsequent peaks in UR. Sectioned left third nerve. M-2 response evoked by mechanical stimulus as UR and PK response as the SCR.

4-1, Four subsequent peaks, with a restored M-2 response, with their first peaks enhanced by depolarizing SCS in the sectioned PK neuron.

4-2, All peaks were inhibited in terms of M-2 response by hyperpolarizing SCS in the sectioned PK neuron.

Calibration: ordinate, $5 \mathrm{~g}$ for $\mathrm{C}$, $50 \mathrm{mV}$ for $\mathrm{M}-2$ and $\mathrm{E}, 5 \mathrm{nA}$ for PK. Abscissa, $5 \mathrm{~s}$ for $\mathrm{C}, 40 \mathrm{~ms}$ for all other traces. See abbreviations in legend to Figure 1.

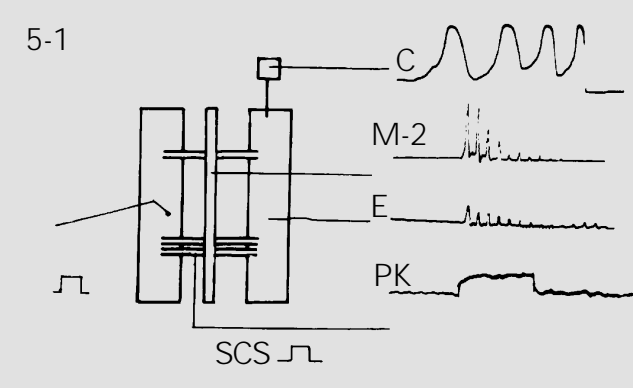

$5-2$

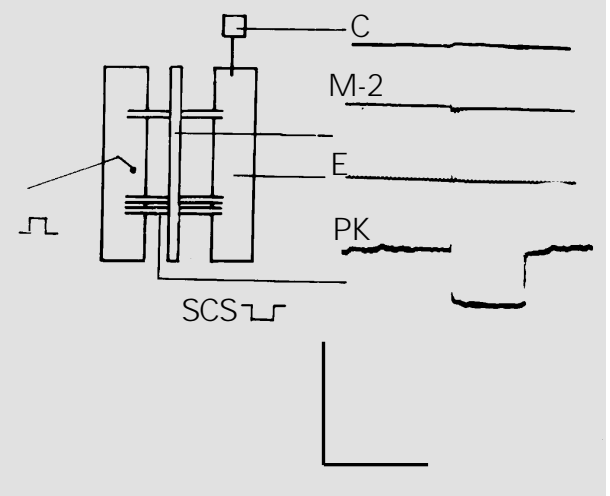

Figure 5 - Summation of the modulatory effects of CS and SCS. Intact left third nerve. M-2 response evoked by a mechanical stimulus as UR. PK response evoked as SCR.

5-1, Increased peak number and enhanced amplitude in M-2 response by depolarizing SCS in the intact PK neuron. Notice increased muscle contraction (C) peaks.

5-2, All peaks were inhibited in terms of the $M-2$ response by hyperpolarizing SCS in the intact PK neuron.

Calibration: ordinate, $5 \mathrm{~g}$ for $\mathrm{C}$, $50 \mathrm{mV}$ for $\mathrm{M}-2$ and $\mathrm{E}, 5 \mathrm{nA}$ for PK. Abscissa, $5 \mathrm{~s}$ for C, $40 \mathrm{~ms}$ for all other traces. See abbreviations in legend to Figure 1. 
hancements, more potential peaks were added to the original five (M-2; Figure 5-1) to a maximal number of 20 with an average number of $11.1 \pm 4.7$ in these 8 preparations. The peak number and amplitude of the electrical responses in the effector muscle cells $(\mathrm{E}$; Figure 5-1) were also enhanced but to a lesser extent than in the $\mathrm{M}-2$ response. The muscle contraction (C; Figure 5-1) was also enhanced until a massive contraction dislodged the microelectrodes.

Hyperpolarizing SCS of more than 4.0 nA inhibited all peaks in the UR (M-2; Figure 5-2) and the muscle cell electrical response (E; Figure 5-2) but did not inhibit the muscle contraction of these preparations completely (C; Figure 5-2).

\section{Morphology of the modulatory neuron}

Only 9 of the 23 modulatory neurons tested showed consistent morphology. Figures 6 and 7 are two examples of these 9 modulatory neurons after filling with Lucifer Yellow-CH. Only one longitudinal band of structure could be focused in the radial dimension (R and PK; Figures 6 and 7, respectively). Its lateral dimensional view retained all the morphological features of the known INT neuron (Figure 6C in Ref. 3), i.e., two afferent processes in the third segmental nerve trunk and two bundles of longitudinal processes at the anterior and posterior sides of a central junction. Both bundles are divided into several branches. Each of these branches contains numerous fine processes (LA and LP; Figure 6). The characteristic feature of the morphology of this neuron is revealed when the neuron is viewed in the anteroposterior dimension (AP; Figure 6) at the cross section of a Kästchen (8), where about 20 of these branches are seen distributed on the surface of this Kästchen; hence, the name, "Peri-Kästchen" neuron given in this article. The three-dimensional reconstruction of this neuron was drawn in Figure 6-K. Its relative position with respect to the M-neurons and effector muscle cell is shown in the radial dimension (Figure 7). Comparing the PK neuron to the known intramural, or intermuscular, neurons (5-7) by replicating the histological methods used in these references, it can be seen that these so-called "intramural", or "intermuscular", neurons (dark cells which are actually stained blue by hematoxylin against the lightcolored muscle cells which are actually stained pink by eosin) are distributed both on the surface and inside these three Kästchenen in Figure 8. The neurons on the surface of these Kästchenen may correspond to the longitudinal branches of a single PK neuron identified in AP (Figure 6) instead of many individual neurons assumed in the early works. The neurons inside these Kästchenen are apparently of a separate category because no Lucifer Yellow-CH was diffused into them (AP; Figure 6). We tentatively called them "Intra-Kästchen" (IK) neurons in contrast to the PK neuron.

\section{Discussion}

The purpose of this study was to identify the mechanism of interaction between responses in the main generator neuron of muscle contraction (1) and in a peripheral neuron of this earthworm. The mechanism was identified as facilitation in the experiments described here. The facilitatory effect of the peripheral PK neuron is not exerted directly on the main generator $\mathrm{M}$ (mechanical-stimulus-sensitive) neuron circuit but indirectly via neurons in a P-circuit parallel to the M-circuit (Figure 1). The primary response evoked in the M-neurons by a mechanical stimulus reverberates through this P-circuit, similar to that in its giant axons (9), and reappears in the M-responses as a second peak. The stronger the facilitatory effect of the PK neuron, the more numerous the subsequent peaks that appear in the M-response. Therefore, only the single peaked M-2 (postsynaptic) neuron response can be 


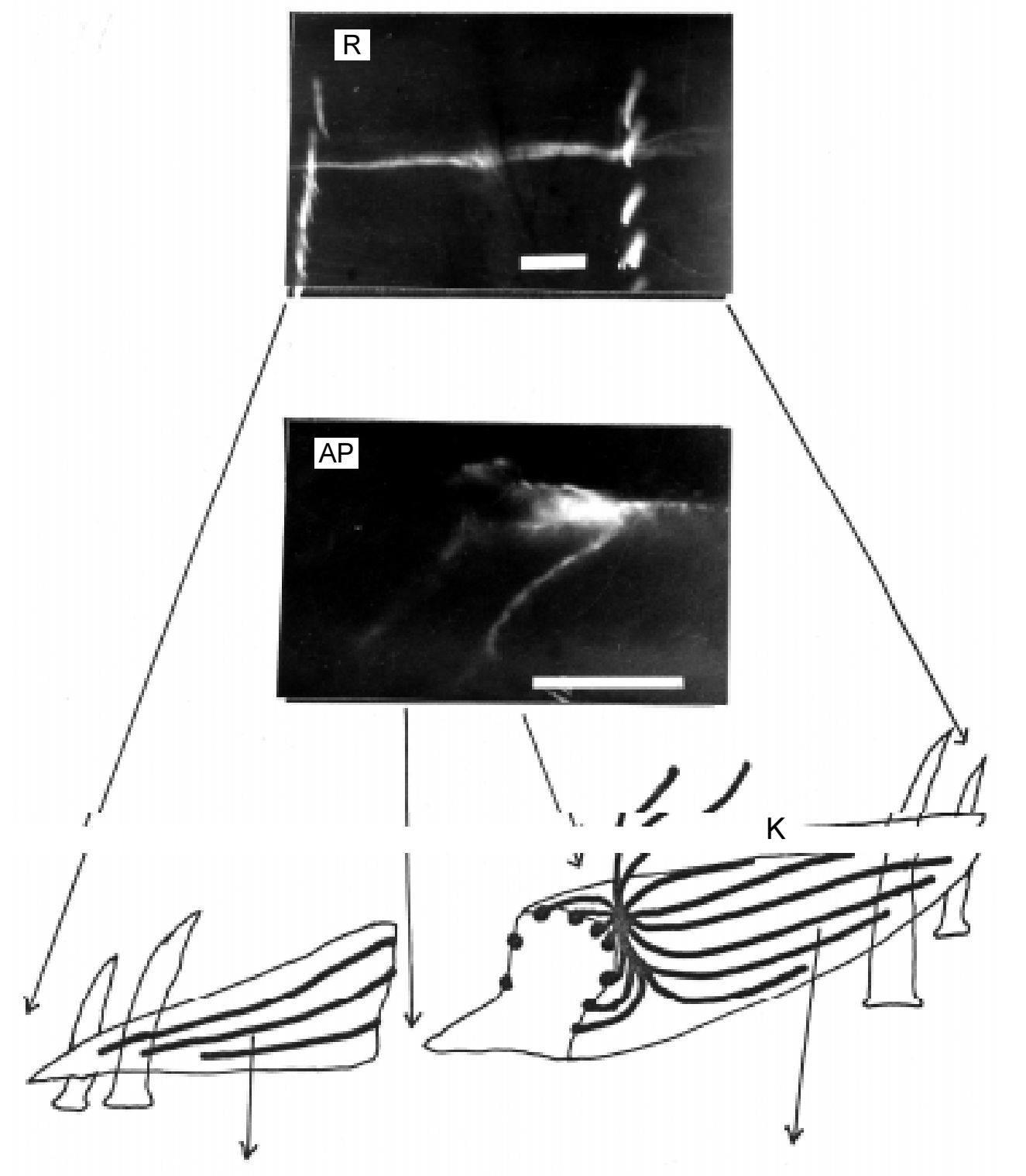

\section{LA}
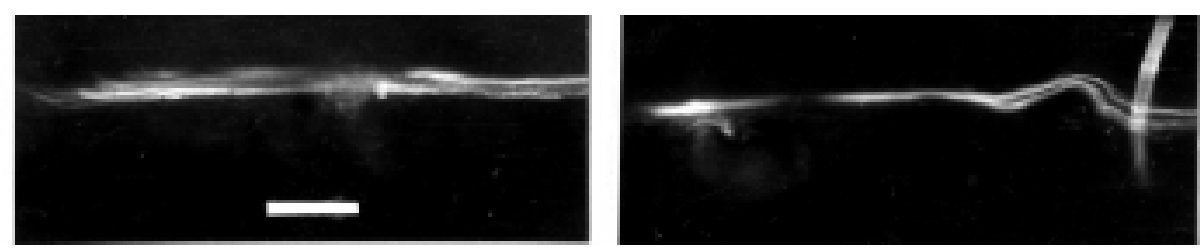

Figure 6 - Morphology of the modulatory neuron. R, Radial dimensional view. The two rows of bright objects are setae with natural fluorescence. AP, Anteroposterior dimensional view at the approximate position shown in Figure 6-K. K, Reconstruction of a Kästchen in three dimensions (not drawn to scale) with PK neuron branches distributed on its surface and the anterior end to the left. The two centripetal processes at the top are inside the third segmental nerve (see Ref. 3). Circular muscle layer and epidermis are visible at the bottom of the drawing. The coelom cavity is shown at the top of the drawing. Four setae can be seen, two at each end of this Kästchen. LA, Lateral dimensional view of part of an anterior branch of the PK neuron; LP, lateral dimensional view of part of a posterior branch of the PK neuron. The calibration bar in all four photographs represents $100 \mu \mathrm{m}$. 
Figure 7 - Anatomical positions of the identified neurons and muscle cells. All cells were filled with Lucifer Yellow-CH and photographed in the radial dimension. The horizontal row of bright objects in the middle of this figure are setae with natural fluorescence. The first pair of segmental nerves is faintly visible above the setae. The second pair of segmental nerves is near the lower edge of this figure. PK, Peri-Kästchen neuron; $M$, mechanical stimulus-sensitive, or generator, neuron slightly unfocused on this plane; $E$, effector muscle cell. Calibration: $100 \mu \mathrm{m}$.

Figure 8 - Comparison of PK neurons to intramural or intermuscular neurons. Anteroposterior dimensional view of a paraffinembedded $10-\mu \mathrm{m}$ thick crosssection of the body of the earthworm, Amynthas hawayanus, showing three Kästchenen. The circular muscle layer and epidermis are visible at the bottom of the figure, and the coelomic cavity at the top. Dark points are neuron-like structures stained blue by hematoxylin. Light points are muscle cells stained pink-red by eosin. Calibration: $100 \mu \mathrm{m}$.

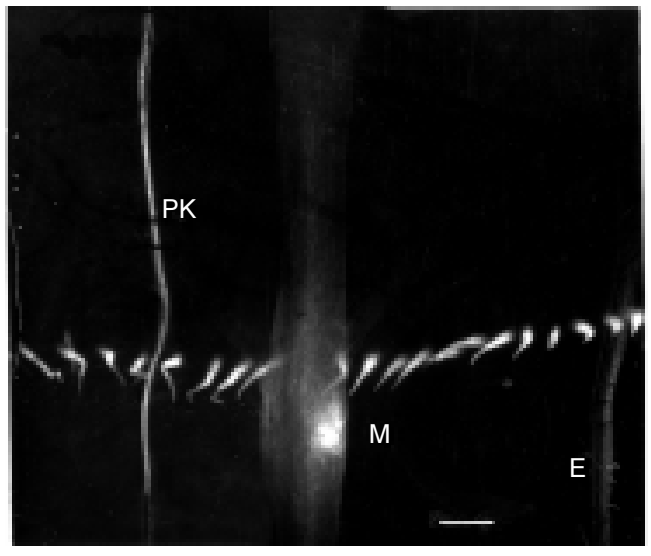

used as the control, or unconditioned, UR, response in this and in the third (4) article because it is free from the facilitatory influence (Figure 2-2). Since the PK response does facilitate this UR, it must be decided if it can be used as a CR in further experiments. A conditioning stimulus, as defined in a conditioning paradigm of the other animals (10-12), modifies the synaptic excitability of the unconditioning neurons persistently. The necessity of coincidence of the US and SCS for their facilitating action on the UR means that this effect is not persistent. Experiments in the third article (4) in this series were designed to find an SCS with a persistent

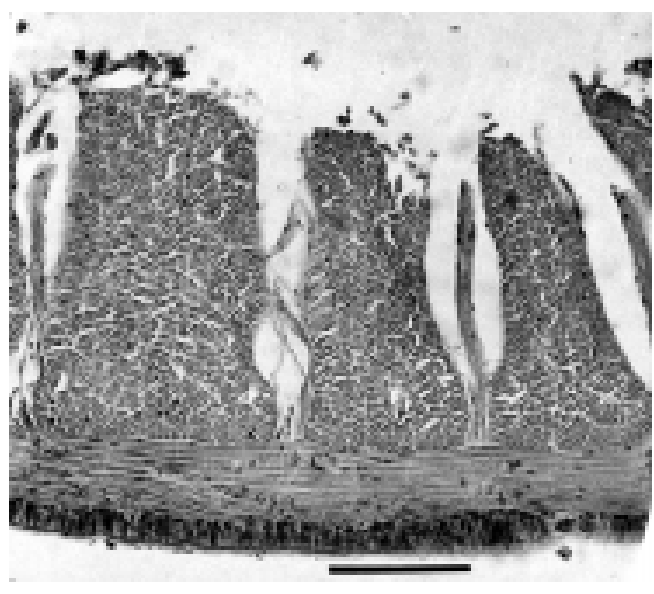
facilitation on the UR in order to be compared to that in the conditioning paradigm of the other animals (10-12).

The morphology of this PK neuron identified by Lucifer Yellow- $\mathrm{CH}$ filling solved a riddle left by histological studies (5-7) where each of these dark-stained structures (Figure 8 ) was counted as an individual intramural neuron. Results in Figures 6, 7 and 8 show that those on the surface of a Kästchen are branches of a single PK neuron while those inside the Kästchen are IK neurons of still unidentified morphology and physiology.

\section{References}

1. Chang YC, Assmé Z, Caffaro ECL \& Bartoszeck AB (1998). Identification of the main generator source of longitudinal muscle contraction in the earthworm ventral nerve cord. Brazilian J oumal of Medical and Biological Research, 31: 12851294.

2. Chang YC \& Assmé $Z$ (1989). Neurônios no circuito reflexo periférico da minhoca, Amynthas hawayanus. Presented at the IV Reunião Anual da Federação de Sociedades de Biologia Experimental, Caxambu, MG, Brasil, 21 (Abstract).

3. Chang $Y C$, Marchioro $M \&$ Assmé $Z$ (1991). Two groups of peripheral afferent neurons in the earthworm reflex arc. Comparative Biochemistry and Physiology, Section A, 100: 563-569.

4. Chang YC, Caffaro ECL, Assmé $Z$ \&
Bartoszeck AB (1998). Persistent attenuation and enhancement of the earthworm main muscle contraction generator response induced by repeated stimulation of a peripheral neuron. Brazilian J ournal of Medical and Biological Research, 31: 1303-1311.

5. Dechant E (1906). Beiträge zur Kenntnis des peripheren Nervensystem des Regenwurmes. Arbeiten von Zoologisches Institut, 16: 361-383.

6. Dawson AB (1920). The intermuscular nerve cells in the earthworm. J ournal of Comparative Neurology, 32: 155-171.

7. Zyeng DH (1930). Distribution of intermuscular nerve cells in the earthworm. Tohoku Imperial University Science Reports, Series 4, 5: 449-466.

8. Hesse R (1894). Zur vergleichenden
Anatomie des Oligochaeten. Zeitschrift für Wissenschaftliche Zoologie, 58: 394-439.

9. Assmé $Z \&$ Chang YC (1990). Decremental propagation of reflex spikes along the giant axons of the earthworm, Amynthas hawayanus. Brazilian J ournal of Medical and Biological Research, 23: 329-332.

10. Carew TJ , Walters ET \& Kandel ER (1981). Classical conditioning in a simple withdrawal reflex in Aplysia californica. J ournal of Neuroscience, 1: 1426-1437.

11. Lukowiak K \& Sahley C (1981). The in vitro classical conditioning of the gill withdrawal reflex of Aplysia californica. Science, 212: 1516-1518.

12. Sastry BR, Goh J W \& Auyeung A (1986). Associative induction of posttetanic and long-term potentiations in CAI neurons of rat hippocampus. Science, 232: 988-990. 